九州大学学術情報リポジトリ

Kyushu University Institutional Repository

\title{
Categorical Representation Theorems of Fuzzy Relations
}

\section{Kawahara, Yasuo}

Department of Informatics Kyushu University

Furusawa, Hitoshi

Department of Informatics Kyushu University

Mori, Masao

Department of Informatics Kyushu University

http://hdl. handle. net/2324/2998

出版情報 : D0I Technical Report. 124, 1996-03-16. Department of Informatics, Kyushu University バージョン：

権利関係 : 


\title{
Categorical Representation Theorems of Fuzzy Relations
}

\author{
Yasuo KAWAHARA, Hitoshi FURUSAWA and Masao MORI*
}

May 16, 1996

\begin{abstract}
This paper provides a notion of Zadeh categories as a categorical structure formed by fuzzy relations with sup-min composition, and proves two representation theorems for Dedekind categories (relation categories) with a unit object analogous to one-point set, and for Zadeh categories without unit objects.
\end{abstract}

Keywords: fuzzy relations, relation algebras, representation theorem, Dedekind categories, Zadeh category.

\section{Introduction}

Since Zadeh's invention the concept of fuzzy sets has been extensively investigated in mathematics, science and engineering. The notion of fuzzy relations is also a basic one in processing fuzzy information in relational structures, see e.g. Pedrycz [10]. Goguen [2] generalized the concepts of fuzzy sets and relations taking values on partially ordered sets. Fuzzy relational equations were initiated and applied to medical models of diagnosis by Sanchez [12].

On the other hand theory of relations, namely relational calculus, has a long history, see $[8,13,14]$ for more details on the history. Almost modern formalizations of relation algebras are affected by the work of Tarski [15]. Mac Lane [7] and Puppe [11] exposed a categorical basis for calculus of additive relations. Freyd and Scedrov [1] developed and summarized categorical relational calculus, which they called allegories. Concerning with applications to the relational theory of graphs and programs, Schmidt and Ströhlein [13] gave a simple proof of a representation theorem for Boolean relation algebras satisfying Tarski rule and point axiom. Also they wrote an excellent text book [14] on relations and graphs with many useful examples in computer science. Also Kawahara and Mizoguchi $[3,6,4]$ developed relational methodology for assertion semantics of programs, theory of graph transformations (or graph grammars) and categorical set theory.

A representation theorem for (homogeneous) fuzzy relation algebras satisfying a point axiom has been given by Kawahara and Furusawa. The aim of the paper is to provide a categorical formalization for fuzzy relations. Fuzzy relations are set-fuctions with values in the unit interval $[0,1]$, that is, functions $R: X \times Y \rightarrow[0,1]$. The set of all such fuzzy relations on $X$ constitutes a fuzzy relation category. An algebra of fuzzy relations are naturally equipped with semi-scalar multiplication by scalars in the unit interval. Also, unlike Boolean relation categories, fuzzy relation categories are not Schröder ones. Instead of Schröder categories we

\footnotetext{
*Department of Informatics, Kyushu University 33, Fukuoka 812-81, Japan.
} 
need to adopt Dedekind categories, named after Jean-Pierre Olivier and Dany Serrato [9], in order to study fuzzy relation categories. This paper is organized as follows:

In section 2 we first recall the fundamentals on relation category, which we will call Dedekind categories following to Jean-Pierre Olivier and Dany Serrato [9], and show a simple representation theorem for Dedekind categories with a unit object satisfying a condition, called the strict point axiom. The section 3 provides a notion and some the basic properties of fuzzy algebras, which are a generalization of algebras formed by set-functions with values in the unit interval $[0,1]$. In section 4 we first state the definition of Zadeh categories as a categorical structure formed by fuzzy relations with sup-min composition. Then some useful properties on point relations, due to G. Schmidt and T. Ströhlein [13], and a point axiom will be introduced to prove a representation theorem for morphisms in Zadeh categories. In particular, the point axiom induces a function assigning a concrete fuzzy relation between the sets of point relations to an abstract relation in Zadeh categories. In section 5 we show a representation theorem for Zadeh categories satisfying a point axiom without the assumption of existence of unit objects. And it is proved that the representation function is a bijection preserving all operations of Zadeh categories. Therefore the representation function turns out to be a functor. However this functor is generally not an isomorphisms of categories since it maps all objects with no points to the empty set. Finally we prove that the representation theorem coincides with the simple representation theorem in section 2, when the Zadeh category has a unit object satisfying the strict point axiom.

\section{Complete Dedekind Categories}

In this section we first recall the fundamentals on relation category, which we will call Dedekind categories following to Jean-Pierre Olivier and Dany Serrato [9], and show a simple representation theorem for Dedekind categories with a unit object satisfying the strict point axiom.

Definition 2.1 A complete Dedekind category $\mathcal{D}$ is a category satisfying the following:

D1. [Complete Distributive Lattice] For all pairs of objects $X$ and $Y$ the homo-set $\mathcal{D}(X, Y)$ consisting of all morphisms of $X$ into $Y$ is a complete distributive lattice with the least morphism $O_{X Y}$ and the greatest morphism $\nabla_{X Y}$.

D2. [Involution] An involution ${ }^{\sharp}: \mathcal{D} \rightarrow \mathcal{D}$ is a monotone contravariant functor. That is, for all morphisms $\alpha, \alpha^{\prime}: X \rightarrow Y, \beta: Y \rightarrow Z$,

(a) $(\alpha \beta)^{\sharp}=\beta^{\sharp} \alpha^{\sharp}$, (b) $\left(\alpha^{\sharp}\right)^{\sharp}=\alpha$, (c) If $\alpha \sqsubseteq \alpha^{\prime}$, then $\alpha^{\sharp} \sqsubseteq \alpha^{\prime \prime}$.

D3. [Dedekind Formula] For all morphisms $\alpha: X-Y, \beta: Y \rightarrow Z$ and $\gamma: X \rightarrow Z$ the Dedekind formula $\alpha \beta \sqcap \gamma \sqsubseteq \alpha\left(\beta \sqcap \alpha^{\sharp} \gamma\right)$ holds.

D4. [Quotient Morphisms] For all morphsms $\beta: Y \rightarrow Z$ and $\gamma: X \rightarrow Z$ a quotient morphism $\gamma \div \beta: X-Y$ is a morphism such that $\alpha \beta \sqsubseteq \gamma$ if and only if $\alpha \sqsubseteq \gamma \div \beta$ for all morphisms $\alpha: X \rightarrow Y$.

Note that complete distributive lattices are equivalent to complete Brouwerian lattices or complete Heyting algebras.

Throughout the section all discussions will be done in a fixed complete Dedekind category $\mathcal{D}$. We denote the identity morphism on an object $X$ of $\mathcal{D}$ by $\mathrm{id}_{X}$. The greatest morphism $\nabla_{X Y}$ is called the universal morphism and the least morphism $O_{X Y}$ the zero morphism. A morphism is nonzero if it is not equal to the zero morphism.

Proposition 2.2 Let $\alpha, \alpha^{\prime}: X \rightarrow Y$ and $\beta, \beta^{\prime}: Y \rightarrow Z$ be morphisms in $\mathcal{D}$.

(a) $O_{X Y}^{\sharp}=O_{Y X}, \nabla_{X Y}^{\sharp}=\nabla_{Y X}$ and $\mathrm{id}_{X}^{\sharp}=\mathrm{id}_{X}$. 
(b) $\nabla_{X X} \nabla_{X Y}=\nabla_{X Y} \nabla_{Y Y}=\nabla_{X Y}$.

(c) $(\alpha \sqcup \beta)^{\sharp}=\alpha^{\sharp} \sqcup \beta^{\sharp}$ and $(\alpha \sqcap \beta)^{\sharp}=\alpha^{\sharp} \sqcap \beta^{\sharp}$.

(d) $O_{X Y} \beta=O_{X Z}$ and $\alpha O_{Y Z}=O_{X Z}$.

(e) $\left(\sqcup_{\lambda} \alpha_{\lambda}\right) \beta=\sqcup_{\lambda} \alpha_{\lambda} \beta$.

(f) If $\alpha \sqsubseteq \alpha^{\prime}$ and $\beta \sqsubseteq \beta^{\prime}$, then $\alpha \beta \sqsubseteq \alpha^{\prime} \beta^{\prime}$.

(g) If $\alpha \sqcup \alpha^{\prime}=\nabla_{X Y}, \alpha \sqcap \alpha^{\prime}=O_{X Y}$ and $\nabla_{X X} \alpha=\alpha$, then $\nabla_{X X} \alpha^{\prime}=\alpha^{\prime}$.

(h) If $\operatorname{id}_{X} \sqsubseteq \alpha \alpha^{\sharp}$ and $\alpha^{\prime \sharp} \alpha^{\prime} \sqsubseteq \mathrm{id}_{Y}$, then $\alpha \sqsubseteq \alpha^{\prime}$ implies $\alpha=\alpha^{\prime}$.

(i) If $\alpha^{\sharp} \alpha \sqsubseteq \mathrm{id}_{Y}$, then $\alpha\left(\beta \sqcap \beta^{\prime}\right)=\alpha \beta \sqcap \alpha \beta^{\prime}$.

(j) If $u \sqsubseteq \mathrm{id}_{X}$ and $v \sqsubseteq \mathrm{id}_{X}$, then $u^{\sharp}=u u=u$ and $u v=u \sqcap v$.

Proof. (a) $O_{X Y}^{\sharp} \sqsubseteq O_{Y X}^{\sharp \sharp}=O_{Y X}$ since $O_{Y X} \sqsubseteq O_{X Y}^{\sharp}$, and $\nabla_{Y X}=\nabla_{Y X}^{\sharp \sharp} \sqsubseteq \nabla_{X Y}^{\sharp}$ since $\nabla_{X Y}^{\sharp} \sqsubseteq$ $\nabla_{Y X}$, and $\mathrm{id}_{X}^{\sharp}=\mathrm{id}_{X}^{\sharp} \mathrm{id}_{X}=\mathrm{id}_{X}^{\sharp} \mathrm{id}_{X}^{\sharp \sharp}=\left(\mathrm{id}_{X}^{\sharp} \mathrm{id}_{X}\right)^{\sharp}=\mathrm{id}_{X}^{\sharp \sharp}=\mathrm{id}{ }_{X}$. (b) Immediate from $\nabla_{X Y}=$ $\operatorname{id}_{X} \nabla_{X Y} \sqsubseteq \nabla_{X X} \nabla_{X Y}$. (c) First note that $\alpha^{\sharp} \sqcup \beta^{\sharp} \sqsubseteq(\alpha \sqcup \beta)^{\sharp}$. Hence $\alpha \sqcup \beta=\alpha^{\sharp \sharp} \sqcup \beta^{\sharp \sharp} \sqsubseteq\left(\alpha^{\sharp} \sqcup \beta^{\sharp}\right)^{\sharp}$ and $(\alpha \sqcup \beta)^{\sharp} \sqsubseteq\left(\alpha^{\sharp} \sqcup \beta^{\sharp}\right)^{\sharp \sharp}=\alpha^{\sharp} \sqcup \beta^{\sharp}$. (d) It is trivial that $O_{X Y} \sqsubseteq O_{X Z} \div \beta$. Hence $O_{X Y} \beta \sqsubseteq O_{X Z}$ by Z3. Using this result we have $\alpha O_{Y Z}=\left(O_{Y Z}^{\sharp} \alpha^{\sharp}\right)^{\sharp}=\left(O_{Z Y} \alpha^{\sharp}\right)^{\sharp}=O_{Z X}^{\sharp}=O_{X Z}$. (e) It follows from $\left(\sqcup_{\lambda} \alpha_{\lambda}\right) \beta \sqsubseteq \gamma \Longleftrightarrow \sqcup_{\lambda} \alpha_{\lambda} \sqsubseteq \gamma \div \beta \Longleftrightarrow \forall \lambda: \alpha_{\lambda} \sqsubseteq \gamma \div \beta \Longleftrightarrow \forall \lambda: \alpha_{\lambda} \beta \sqsubseteq \gamma \Longleftrightarrow \sqcup_{\lambda} \alpha_{\lambda} \beta \sqsubseteq \gamma$. (f) If $\beta \sqsubseteq \beta^{\prime}$, then $\alpha \beta \sqsubseteq \alpha \beta \sqcup \alpha \beta^{\prime}=\alpha\left(\beta \sqcup \beta^{\prime}\right)=\alpha \beta^{\prime}$ by (d). (g) Note that $\alpha^{\prime}=\mathrm{id}_{X} \alpha^{\prime} \sqsubseteq \nabla_{X X} \alpha^{\prime}$, and $\nabla_{X X} \alpha^{\prime} \sqcap \alpha \sqsubseteq \nabla_{X X}\left(\alpha^{\prime} \sqcap \nabla_{X X}^{\sharp} \alpha\right)=\nabla_{X X}\left(\alpha^{\prime} \sqcap \nabla_{X X} \alpha\right)=\nabla_{X X}\left(\alpha^{\prime} \sqcap \alpha\right)=\nabla_{X X} O_{X Y}=O_{X Y}$. Hence $\nabla_{X X} \alpha^{\prime}=\nabla_{X X} \alpha^{\prime} \sqcap \nabla_{X Y}=\nabla_{X X} \alpha^{\prime} \sqcap\left(\alpha \sqcup \alpha^{\prime}\right)=\left(\nabla_{X X} \alpha^{\prime} \sqcap \alpha\right) \sqcup\left(\nabla_{X X} \alpha^{\prime} \sqcap \alpha^{\prime}\right)=\alpha^{\prime}$.

The statement (b) in the last proposition indicates that if $\nabla_{X Y} \neq O_{X Y}$ then both of $X$ and $Y$ are nonempty.

Proposition 2.3 Let $\alpha: X \rightarrow Y$ be a morphism such that $\nabla_{X X} \alpha=\alpha$. Then the following three conditions are equivalent: (a) $\mathrm{id}_{X} \sqsubseteq \alpha \alpha^{\sharp}$, (b) $\nabla_{X X}=\alpha \alpha^{\sharp}$, (c) $\nabla_{X X}=\alpha \nabla_{Y X}$.

Proof. (a) $\Rightarrow$ (b) If id ${ }_{X} \sqsubseteq \alpha \alpha^{\sharp}$, then $\nabla_{X X}=\nabla_{X X}$ id $_{X} \sqsubseteq \nabla_{X X} \alpha \alpha^{\sharp}=\alpha \alpha^{\sharp}$. (b) $\Rightarrow$ (c) If $\nabla_{X X}=\alpha \alpha^{\sharp}$, then $\nabla_{X X}=\alpha \alpha^{\sharp} \sqsubseteq \alpha \nabla_{Y X}$. (c) $\Rightarrow$ (a) If $\nabla_{X X}=\alpha \nabla_{Y X}$, then $\mathrm{id}_{X}=\mathrm{id}{ }_{X} \sqcap \nabla_{X X}=$ $\operatorname{id}_{X} \sqcap \alpha \nabla_{Y X} \sqsubseteq \alpha\left(\alpha^{\sharp} \mathrm{id}_{X} \sqcap \nabla_{Y X}\right)=\alpha \alpha^{\sharp}$.

We now introduce three notions of unit objects, $I$-crisp relations and $I$-points in Dedekind categories. A unit object is an abstrction of the notion of singleton (or one-point) sets. Two distinct univalent and total relation from a unit object are not mutually disjoint. To this end the notion of $I$-points are in addition assumed to be $I$-crisp.

Definition 2.4 Let $\mathcal{D}$ be a complete Dedekind category.

(a) An object $I$ in $\mathcal{D}$ is called a unit object if $O_{I I} \neq \mathrm{id}_{I}=\nabla_{I I}$.

(b) A morphism $\tau: I \rightarrow X$ is $I$-crisp if $u \rho \sqsubseteq \tau$ implies $\rho \sqsubseteq \tau$ for a nonzero morphism $u: I \rightarrow I$ and a morphism $\rho: I \rightarrow X$.

(c) An $I$-point $p$ of $X$ is an $I$-crisp morphism $p: I-X$ such that $p^{\sharp} p \sqsubseteq \mathrm{id}_{X}, \mathrm{id}_{I}=p p^{\sharp}$.

(d) A unit object $I$ satisfies the strict point axiom if $\sqcup_{p \in \xi(X)} p^{\sharp} p=\operatorname{id}_{X}$ for all objects $X$, where $\xi(X)$ denotes the set of all $I$-points of $X$. 
Note that an $I$-point $p$ of $X$ is nonempty from its totality condition $\mathrm{id}_{I}=p p^{\sharp}$. Thus if an object has at least one $I$-point then it is nonempty.

Proposition 2.5 Let I be a unit object in $\mathcal{D}$.

(a) If $\tau \sqsubseteq p$ for an $I$-point $p: I \rightarrow X$ and a morphism $\tau: I \rightarrow X$, then $\tau=$ wp for a unique morphism $w: I \rightarrow I$.

(b) If $p_{0} \neq p_{1}$ for $I$-points $p_{0}, p_{1}: I-X$, then $p_{0} \sqcap p_{1}=O_{I X}$ and $p_{0} p_{1}^{\sharp}=O_{I I}$.

(c) $(u \sqcap v) \tau=u \tau \sqcap v \tau$ for morphisms $u, v: I \rightarrow I$ and $\tau: I \rightarrow X$.

Proof. (a) $\tau p^{\sharp} p \sqsubseteq \tau=\tau \sqcap p \sqsubseteq\left(\tau p^{\sharp} \sqcap \mathrm{id}_{I}\right) p=\tau p^{\sharp} p$ by D3. (b) First we show that if $p_{0} \sqcap p_{1} \neq O_{I X}$ then $p_{0}=p_{1}$. As $p_{0} \sqcap p_{1} \sqsubseteq p_{0}$, by (a) there is a unique $w: I-I$ such that $p_{0} \sqcap p_{1}=w p_{0}$. If $p_{0} \sqcap p_{1} \neq O_{I X}$, then $w \neq O_{I I}$ and so $p_{0} \sqsubseteq p_{1}$, because $w p_{0} \sqsubseteq p_{1}$ and $p_{1}$ is $I$-crisp. Finally if $p_{0} \sqcap p_{1}=O_{I X}$, then $p_{0} p_{1}^{\sharp}=p_{0} p_{1}^{\sharp} \sqcap \mathrm{id} d_{I} \sqsubseteq\left(p_{0} \sqcap \mathrm{id} p_{1}\right) p_{1}^{\sharp}=\left(p_{0} \sqcap p_{1}\right) p_{1}^{\sharp}=O_{I I}$ by D3 and $2.2(\mathrm{a})$. (c) $(u \sqcap v) \tau \sqsubseteq u \tau \sqcap v \tau \sqsubseteq\left(u \sqcap v \tau \tau^{\sharp}\right) \tau \sqsubseteq(u \sqcap v) \tau$.

Set $L=\mathcal{D}(I, I)$ and let $\alpha: X \rightarrow Y$ be a morphism. Then $L$ is a complete disributive lattice by D1. A function $\xi(\alpha): \xi(X) \times \xi(Y) \rightarrow L$ assgining $\xi(\alpha)(p, q)=p \alpha q^{\sharp}: I \rightarrow I$ to a pair of $I$-points $p: I \rightarrow X$ and $q: I \rightarrow Y$, gives an $L$-fuzzy relation of $\xi(X)$ into $\xi(Y)$ in the sense of [2]. The infimum (the greatest lower bound) and the supremum (the least upper bound) of $L$-fuzzy relations will be denoted by the symbols $\cap$ and $\cup$. We write the category of sets and $L$-fuzzy relations as $\mathbf{R e l}_{L}$.

Theorem 2.6 (Representation Theorem) Let I be a unit object satisfying the strict point axiom. Then every morphism $\alpha: X \rightarrow Y$ has a unique representation

$$
\alpha=\sqcup_{p \in \xi(X)} \sqcup_{q \in \xi(Y)} p^{\sharp} \xi(\alpha)(p, q) q,
$$

where $\xi(\alpha)(p, q)=p \alpha q^{\sharp}$ for $I$-points $p: I \rightarrow X$ and $q: I \rightarrow Y$.

Proof. Since $\operatorname{id}_{X}=\sqcup_{p \in \xi(X)} p^{\sharp} p$ and $\operatorname{id}_{Y}=\sqcup_{q \in \xi(Y)} q^{\sharp} q$ by the strict point axiom we have

$$
\begin{aligned}
\alpha & =\operatorname{id}_{X} \alpha \operatorname{id}_{Y} \\
& =\left(\sqcup_{p \in \xi(X)} p^{\sharp} p\right) \alpha\left(\sqcup_{q \in \xi(Y)} q^{\sharp} q\right) \\
& =\sqcup_{p \in \xi(X)} \sqcup_{q \in \xi(Y)} p^{\sharp} p \alpha q^{\sharp} q \\
& =\sqcup_{p \in \xi(X)} \sqcup_{q \in \xi(Y)} p^{\sharp} \xi(\alpha)(p, q) q .
\end{aligned}
$$

Finally we show the uniqueness of the representation. Assume $\alpha=\sqcup_{p \in \xi(X)} \sqcup_{q \in \xi(Y)} p^{\sharp} k_{p, q} q$. Then for all $p_{0} \in \xi(X), q_{0} \in \xi(Y)$ we have $\xi(\alpha)\left(p_{0}, q_{0}\right)=p_{0} \alpha q_{0}^{\sharp}=\sqcup_{p \in \xi(X)} \sqcup_{q \in \xi(Y)} p_{0} p^{\sharp} k_{p, q} q q_{0}^{\sharp}=k_{p_{0}, q_{0}}$ by $2.5(b)$.

Corollary 2.7 Let I be a unit object satisfying the strict point axiom.

(a) The function $\xi: \mathcal{D}(X, Y) \rightarrow \operatorname{Rel}_{L}(\xi(X), \xi(Y))$ is bijective.

(b) $\xi\left(O_{X Y}\right)=O_{\xi(X) \xi(Y)}, \xi\left(\nabla_{X Y}\right)=\nabla_{\xi(X) \xi(Y)}$ and $\xi\left(\mathrm{id}_{X}\right)=\mathrm{id}_{\xi(X)}$,

(c) $\xi\left(\alpha \sqcup \alpha^{\prime}\right)=\xi(\alpha) \cup \xi\left(\alpha^{\prime}\right)$ and $\xi\left(\alpha \sqcap \alpha^{\prime}\right)=\xi(\alpha) \cap \xi\left(\alpha^{\prime}\right)$,

(d) $\xi\left(\alpha^{\sharp}\right)=\xi(\alpha)^{\sharp}$, 
(e) $\xi(\alpha \beta)=\xi(\alpha) \xi(\beta)$.

Proof. (b) It is immediate that $\xi\left(O_{X Y}\right)(p, q)=p O_{X Y} q^{\sharp}=O_{I I}$. It follows from id $\mathrm{d}_{I}=$ $\operatorname{id}_{I} \mathrm{id}_{I}=p p^{\sharp} q q^{\sharp} \sqsubseteq p \nabla_{X Y} q^{\sharp}$ that $\xi\left(\nabla_{X Y}\right)(p, q)=\mathrm{id}_{I}$, and $\xi\left(\operatorname{id}_{X}\right)(p, q)=p \operatorname{id}_{X} q^{\sharp}=p q^{\sharp}$. (c) $\xi\left(\alpha \sqcup \alpha^{\prime}\right)(p, q)=p\left(\alpha \sqcup \alpha^{\prime}\right) q^{\sharp}=p \alpha q^{\sharp} \sqcup p \alpha^{\prime} q^{\sharp}=\left[\xi(\alpha) \cup \xi\left(\alpha^{\prime}\right)\right](p, q)$ and $\xi\left(\alpha \sqcap \alpha^{\prime}\right)(p, q)=$ $\chi\left(\alpha \sqcap \alpha^{\prime}\right) q^{\sharp}=p \alpha q^{\sharp} \sqcap p \alpha^{\prime} q^{\sharp}=\left[\xi(\alpha) \cap \xi\left(\alpha^{\prime}\right)\right](p, q)$. (d) $\xi\left(\alpha^{\sharp}\right)(q, p)=q \alpha^{\sharp} p^{\sharp}=\left(p \alpha q^{\sharp}\right)^{\sharp}=p \alpha q^{\sharp}=$ $\xi(\alpha)(p, q)=\xi(\alpha)^{\sharp}(q, p)$. (e) $\xi(\alpha \beta)(p, r)=p \alpha \beta r^{\sharp}=\sqcup_{q \in \xi(Y)} p \alpha q^{\sharp} q \beta r^{\sharp}=\sqcup_{q \in \xi(Y)}\left(p \alpha q^{\sharp} \sqcap q \beta r^{\sharp}\right)=$ $[\xi(\alpha) \xi(\beta)](p, r)$ since $p \alpha q^{\sharp}, q \beta r^{\sharp} \sqsubseteq \mathrm{id}_{I}$.

From the proof of $2.7(\mathrm{~b})$ it is easy to see that $\nabla_{X Y} \neq O_{X Y}$ for all nonempty objects $X$ and $Y$ (Cf. a condition P1 in Definition 4.4) if $\mathcal{D}$ has a unit object $I$ satisfying the strict point axiom.

Corollary 2.8 Let I be a unit object satisfying the strict point axiom. Then the following holds:

(a) $\sqcup_{p \in \xi(X)} p=\nabla_{I X}$,

(b) $\nabla_{X Y}=\nabla_{X I} \nabla_{I Y}$,

(c) If $Y$ has at least one I-point, then $\nabla_{X Y} \nabla_{Y Z}=\nabla_{X Z}$.

Proof. (a) For each $I$-point $p: I-X$ we have $p \nabla_{X I}=\nabla_{I I}=\mathrm{id}_{I}$ by $2.3(\mathrm{c})$. Hence using the strict point axiom $\nabla_{I X}=\nabla_{I X} \mathrm{id}_{X}=\sqcup_{p \in \xi(X)} \nabla_{I X} p^{\sharp} p=\sqcup_{p \in \xi(X)}\left(p \nabla_{X I}\right)^{\sharp} p=\sqcup_{p \in \xi(X)} p$. (b) By $2.7(b)$ we have $\xi\left(\nabla_{X Y}\right)(p, q)=\mathrm{id}_{I}$ and so

$$
\nabla_{X Y}=\sqcup_{p \in \xi(X)} \sqcup_{q \in \xi(Y)} p^{\sharp} q=\left(\sqcup_{p \in \xi(X)} p\right)^{\sharp}\left(\sqcup_{q \in \xi(Y)} q\right)=\nabla_{I X}^{\sharp} \nabla_{I Y}
$$

by the representation theorem 2.6 and (a). (c) Let $q$ be an $I$-point of $Y$. Then $\nabla_{I X}=q q^{\sharp} \nabla_{I X} \sqsubseteq$ $q \nabla_{Y X}$ and $\nabla_{I Z}=q q^{\sharp} \nabla_{I Z} \sqsubseteq q \nabla_{Y Z}$. Hence by using (b) $\nabla_{X Z}=\nabla_{I X}^{\sharp} \nabla_{I Z} \sqsubseteq\left(q \nabla_{Y X}\right)^{\sharp} q \nabla_{Y Z}=$ $\nabla_{X Y} q^{\sharp} q \nabla_{Y Z} \sqsubseteq \nabla_{X Y} \nabla_{Y Z} \cdot$

As a result we have proved that a Dedekind category with a unit object satisfying the strict point axiom is equivalent to a subcategory of a category of $L$-fuzzy relations.

\section{$3 \quad$ Fuzzy Algebras}

This section introduces a notion of fuzzy algebras as a mathematical strucutre formed by fuzzy sets, and some basic properties of fuzzy algebras are described. Roughly speaking fuzzy algebras are complete distributive lattices with semi-multiplcation and semi-Boolean property. Throughout of the paper the unit interval of reals $k$ with $0 \leq k \leq 1$ will be denoted by a symbol $[0,1]$ and reals $k \in[0,1]$ will be called scalars.

Definition 3.1 A fuzzy algebra $\mathcal{F}=(\mathcal{F}, \sqsubseteq, \sqcup, \sqcap, \cdot, O, \nabla)$ is an algebraic structure over a nonempty set $\mathcal{F}$ satisfying the following:

F1. [Complete Distributive Lattice] A hexad $(\mathcal{F}, \sqsubseteq, \sqcup, \sqcap, O, \nabla)$ is a complete distributive lattice with the least element $O$ and the greatest element $\nabla$.

F2. [Semi-Scalar Multiplication] An operation $\cdot:[0,1] \times \mathcal{F} \rightarrow \mathcal{F}$ is a semi-scalar multiplication on $\mathcal{F}$. That is,

(a) $0 a=O$ and $1 a=a$, (b) $k\left(k^{\prime} a\right)=\left(k k^{\prime}\right) a$, (c) $k\left(\sqcup_{\lambda} a_{\lambda}\right)=\sqcup_{\lambda} k a_{\lambda}$ and $k\left(\sqcap_{\lambda} a_{\lambda}\right)=\sqcap_{\lambda} k a_{\lambda}$,

(d) $\left(\wedge_{\lambda} k_{\lambda}\right) a=\sqcap_{\lambda} k_{\lambda} a$, (e) If $k a \sqsubseteq k b$ and $k>0$, then $a \sqsubseteq b$. 
(The multiplication $k \cdot a$ of $a \in \mathcal{F}$ by a scalar $k \in[0,1]$ will be written as $k a$, unless confusion occurs.)

F3. [Semi-Boolean Algebra] If $a \sqcap k \nabla=k a$ for all scalars $k$, then there is an element $b$ such that $a \sqcup b=\nabla$ and $a \sqcap b=O$.

Throughout the rest of the section all discussions will be done in a fixed fuzzy algebra $\mathcal{F}$.

Proposition 3.2 Let $a, b$ be elements of a fuzzy algebra $\mathcal{F}$ and $k, k^{\prime}$ scalars.

(a) If $a \sqsubseteq b$, then $k a \sqsubseteq k b$.

(b) If $k \leq k^{\prime}$, then $k a \sqsubseteq k^{\prime} a$. In particular, $k a \sqsubseteq a$ and $k O=O$.

(c) $k a \sqcup k^{\prime} a=\left(k \vee k^{\prime}\right) a$.

(d) If $k \nabla=\nabla$ and $k \neq 1$, then $\nabla=O$.

(e) If $k \nabla=\nabla$ and $\nabla \neq O$, then $k=1$.

(f) If $k \nabla \sqsubseteq k^{\prime} \nabla$ and $\nabla \neq O$, then $k \leq k^{\prime}$.

Proof. (a) If $a \sqsubseteq b$, then $k a=k(a \sqcap b)=k a \sqcap k b \sqsubseteq k b$ by F2(c). (b) If $k \leq k^{\prime}$, then $k a=\left(k \wedge k^{\prime}\right) a=k a \sqcap k^{\prime} a \sqsubseteq k^{\prime} a$ by F2(d). (c) Assume $k \leq k^{\prime}$. Then $k a \sqcup k^{\prime} a=k^{\prime} a=\left(k \vee k^{\prime}\right) a$ by (b). (d) Assume $k \nabla=\nabla$ and $0 \leq k<1$. Then by F2(b) it is trivial that $k^{n} \nabla=\nabla$ for all natural numbers $n$. Hence $\nabla=\Pi_{n \geq 0} k^{n} \nabla=\left(\wedge_{n \geq 0} k^{n}\right) \nabla=0 \nabla=O$ by F2(d) and F2(a). (e) This statement is obviously equivalent to (d). (f) Assume $k \nabla \sqsubseteq k^{\prime} \nabla$ and $k^{\prime}<k$. Then $0 \leq k^{\prime} / k<1$ and $k \nabla \sqsubseteq k\left[\left(k^{\prime} / k\right) \nabla\right]$. Therefore $\nabla \sqsubseteq\left(k^{\prime} / k\right) \nabla$ by $\mathrm{F} 2(\mathrm{e})$ and so $\nabla=O$ by $(\mathrm{d})$.

Following to [5] the concept of crisp elements in fuzzy algebras is defined as follows:

Definition 3.3 An element $a$ of a fuzzy algebra $\mathcal{F}$ is crisp if $a \sqcap k \nabla=k a$ for all scalars $k \in[0,1]$.

In the above definition of crisp elements of fuzzy algebras it is trivial that $k a \sqsubseteq a \sqcap k \nabla$ by $3.2(\mathrm{a})$ and $3.2(\mathrm{~b})$. Note that $\nabla \sqcap k \nabla=k \nabla$ from $k \nabla \sqsubseteq \nabla$ by F1(b). This means that the universal element $\nabla$ is crisp. Also the zero element $O$ is clearly crisp.

Proposition 3.4 Let $a, b$ be elements of $\mathcal{F}$ and $k$ a scalar.

(a) If $a$ and $b$ are crisp, then so are $a \sqcup b$ and $a \sqcap b$.

(b) If $a \sqcup b=\nabla$ and $a \sqcap b=O$, then both of $a$ and $b$ are crisp.

(c) If $b$ is crisp and $k a \sqsubseteq b$ for $k>0$, then $a \sqsubseteq b$.

Proof. (a) Let $a$ and $b$ be crisp. Then $(a \sqcup b) \sqcap k \nabla=(a \sqcap k \nabla) \sqcup(b \sqcap k \nabla)=k a \sqcup k b=k(a \sqcup b)$ by $\mathrm{F} 1$ and $\mathrm{F} 2(\mathrm{c}) . \quad(a \sqcap b) \sqcap k \nabla=(a \sqcap k \nabla) \sqcap(b \sqcap k \nabla)=k a \sqcap k b=k(a \sqcap b)$ by $\mathrm{F} 2(\mathrm{c})$. (b) Assume $a \sqcup b=\nabla$ and $a \sqcap b=O$. First by 3.2(b) $k \alpha \sqsubseteq \alpha$ and $\alpha \sqcap k \beta=O$. Then $\alpha \sqcap k \nabla=\alpha \sqcap k(\alpha \sqcup \beta)=(\alpha \sqcap k \alpha) \sqcup(\alpha \sqcap k \beta)=k \alpha$ by F2(c) and F1. (c) Note that $k a \sqsubseteq k \nabla$ by $3.2(\mathrm{a})$. As $b$ is crisp we have $k a \sqsubseteq b \sqcap k \nabla=k b$. Hence $a \sqsubseteq b$ by F2(e).

From the last proposition 3.4(a) and 3.4(b) it is immediate that the set of all crisp elements in a fuzzy algebra $\mathcal{F}$ forms a Boolean algebra. 


\section{Zadeh Categories}

In this section we first state the definition of Zadeh categories as a categorical structure formed by fuzzy relations with sup-min composition. Then some useful properties on point relations, due to G. Schmidt and T. Ströhlein [13], and a point axiom will be introduced to prove a representation theorem for morphisms in Zadeh categories. In particular, the point axiom induces a function assigning a concrete fuzzy relation between the sets of point relations to an abstract relation in Zadeh categories.

Definition 4.1 A Zadeh category $\mathcal{Z}$ is a complete Dedekind category satisfying the following: Z1. [Fuzzy Algebra] For all objects $X$ and $Y$ the homo-set $\mathcal{Z}(X, Y)$ consisting of all morphisms of $X$ into $Y$ is a fuzzy algebra. Its fuzzy algebra structure will be denoted by

$$
\mathcal{Z}(X, Y)=\left(\mathcal{Z}(X, Y), \sqsubseteq, \sqcup, \sqcap, \cdot, O_{X Y}, \nabla_{X Y}\right) .
$$

Z2. [Compatibility with Semi-Scalar Multiplication]

(a) $k(\alpha \beta)=(k \alpha)(k \beta),\left(\right.$ b) $(k \alpha)^{\sharp}=k \alpha^{\sharp},(\mathrm{c})(k \alpha) \beta=(k \alpha)\left(\beta \sqcap k \nabla_{Y Z}\right)$ for morphisms $\alpha: X \rightarrow Y, \beta: Y \rightarrow Z$, and a scalar $k$.

Throughout the rest of the paper all discussions will be done in a fixed Zadeh category $\mathcal{Z}$.

Proposition 4.2 Let $\alpha: X \rightarrow Y$ and $\beta: Y-Z$ be morphisms and $k$ a scalar.

(a) $(k \alpha) \beta \sqsubseteq k \nabla_{X Z}$ and $\alpha(k \beta) \sqsubseteq k \nabla_{X Z}$.

(b) If $\beta$ is crisp, then $(k \alpha) \beta=k(\alpha \beta)$. (If $\alpha$ is crisp, then $\alpha(k \beta)=k(\alpha \beta)$ ).

(c) If $\alpha$ and $\beta$ are crisp, then so are $\alpha^{\sharp}$ and $\alpha \beta$.

(d) The identity morphism $\mathrm{id}_{X}$ is crisp.

Proof. (a) From Z2(c), Z2(a) it follows that $(k \alpha) \beta=(k \alpha)\left(\beta \sqcap k \nabla_{Y Z}\right) \sqsubseteq(k \alpha)\left(k \nabla_{Y Z}\right)=$ $k\left(\alpha \nabla_{Y Z}\right) \sqsubseteq k \nabla_{X Z}$. (b) Assume that $\beta$ is crisp. Then $(k \alpha) \beta=(k \alpha)\left(\beta \sqcap k \nabla_{Y Z}\right)=(k \alpha)(k \beta)=$ $k(\alpha \beta)$ by Z2(c) and Z2(a). (c) $\alpha^{\sharp} \sqcap k \nabla_{Y X}=\left(\alpha \sqcap k \nabla_{X Y}\right)^{\sharp}=(k \alpha)^{\sharp}=k \alpha^{\sharp}$ by $2.2(\mathrm{a})$, $2.2(\mathrm{~b})$ and Z2(b). $\alpha \beta \sqcap k \nabla_{X Z} \sqsubseteq \alpha\left[\beta \sqcap \alpha^{\sharp}\left(k \nabla_{X Z}\right)\right] \sqsubseteq \alpha\left(\beta \sqcap k \nabla_{Y Z}\right)=\alpha(k \beta)=k(\alpha \beta)$ by D3, 3.2(a), Z2(c) and (b). (d) $\mathrm{id}_{X} \sqcap k \nabla_{X X}=\mathrm{id}_{X} \sqcap k\left(\mathrm{id}_{X} \nabla_{X X}\right)=\mathrm{id}_{X} \sqcap\left(k \mathrm{id}_{X}\right) \nabla_{X X} \sqsubseteq$ $\left(\operatorname{kid}_{X}\right)\left[\left(\operatorname{kid}_{X}\right)^{\sharp i d_{X}} \sqcap \nabla_{X X}\right]=k \operatorname{id}_{X}$.

In view of $[5,13]$ the concept of points in Zadeh categories is defined as follows:

Definition 4.3 A point $x$ of an object $X$ is a crisp morphism $x: X \rightarrow X$ such that $x^{\sharp} x \sqsubseteq \mathrm{id}{ }_{X}$, $\operatorname{id}_{X} \sqsubseteq x x^{\sharp}$ and $\nabla_{X X} x=x$.

Note that a point $x$ of $X$ is automatically nonzero from the totality $\mathrm{id}_{X} \sqsubseteq x x^{\sharp}$ if $X$ is nonempty. An empty object has no points because points are nonzero by the definition.

By making use of the last definition of points in Zadeh categories we add the following axiom:

Definition 4.4 A Zadeh category $\mathcal{Z}$ satisfies the point axiom if:

$\mathrm{P} 1$. For all objects $X$ and $Y$ the universal morphism $\nabla_{X Y}$ is nonzero (that is, $\nabla_{X Y} \neq O_{X Y}$ ).

P2. For a nonzero morphism $\alpha: X-Y$ there is a scalar $k>0$ and points $x: X-X$ and $y: Y \rightarrow Y$ such that $x \alpha \sqcap \nabla_{X Y} y=k \nabla_{X Y} y$. 
In what follows we assume that a fixed Zadeh category $\mathcal{Z}$ satisfies the point axiom above.

Proposition 4.5 Let $x_{0}, x_{1}: X \rightarrow X, y, y_{0}, y_{1}: Y \rightarrow Y$ points, and $k$ and $k^{\prime}$ scalars. Then the following holds:

(a) If $x_{0} \neq x_{1}$, then $x_{0} \sqcap x_{1}=O_{X X}$ and $x_{0} x_{1}^{\sharp}=O_{X X}$.

(b) If $\nabla_{X Y} y_{0} \sqsubseteq \nabla_{X Y} y_{1}$, then $y_{0}=y_{1}$.

(c) If $x_{0}^{\sharp} \nabla_{X Y} y_{0} \sqsubseteq x_{1}^{\sharp} \nabla_{X Y} y_{1}$, then $x_{0}=x_{1}$ and $y_{0}=y_{1}$.

(d) If $\alpha$ is a nonzero crisp morphism and $k \alpha \sqsubseteq k^{\prime} \alpha^{\prime}$, then $k \leq k^{\prime}$.

(e) If $\gamma \sqsubseteq \nabla_{X Y} y$ and $\nabla_{X X} \gamma=\gamma$, then there is a unique scalar $k$ such that $\gamma=k \nabla_{X Y} y$.

Proof. (a) First we show that if $x_{0} \sqcap x_{1} \neq O_{X X}$ then $x_{0}=x_{1}$. Assume that $x_{0} \sqcap x_{1} \neq O_{X X}$. Then by the point axiom there are a scalar $k>0$ and points $z_{0}, z_{1}$ such that $z_{0}\left(x_{0} \sqcap x_{1}\right) \sqcap z_{1}=k z_{1}$. Hence $z_{1} \sqsubseteq z_{0}\left(x_{0} \sqcap x_{1}\right) \sqsubseteq \nabla_{X X} x_{0} \sqcap \nabla_{X X} x_{1}=x_{0} \sqcap x_{1}$. Therefore $x_{0}=z_{1}=x_{1}$ by $2.2(\mathrm{~h})$. Finally, if $x_{0} \sqcap x_{1}=O_{X X}$, then $x_{0} x_{1}^{\sharp}=x_{0} x_{1}^{\sharp} \sqcap \nabla_{X X} \sqsubseteq\left(x_{0} \sqcap \nabla_{X X} x_{1}\right) x_{1}^{\sharp}=\left(x_{0} \sqcap x_{1}\right) x_{1}^{\sharp}=$ $O_{X X}$. (b) Assume that $\nabla_{X Y} y \sqsubseteq \nabla_{X Y} y_{0}$. Then $\nabla_{X Y} \sqsubseteq \nabla_{X Y} y y^{\sharp}=\nabla_{X Y} y_{0} y^{\sharp}$ and so $y_{0} y^{\sharp} \neq$ $O_{Y Y}$ from $\nabla_{X Y} \neq O_{Y Y}$. Hence $y=y_{0}$ by (a). (c) First note that $\nabla_{X Y}=\nabla_{X X}^{\sharp} \nabla_{X Y}=$ $\left(x \nabla_{X X}\right)^{\sharp} \nabla_{X Y}=\nabla_{X X} x^{\sharp} \nabla_{X Y}$ by 2.2(g) and 2.3(c). Assume that $x^{\sharp} \nabla_{X Y} y \sqsubseteq x_{0}^{\sharp} \nabla_{X Y} y_{0}$. Then $\nabla_{X Y} y=\nabla_{X X} x^{\sharp} \nabla_{X Y} y \sqsubseteq \nabla_{X X} x_{0}^{\sharp} \nabla_{X Y} y_{0}=\nabla_{X Y} y_{0}$. Hence $y=y_{0}$ by (b). Similarly $x=x_{0} .(\mathrm{d})$ By the point axiom there are a scalar $h>0$ and points $x, y$ such that $x \alpha \sqcap \nabla_{X Y} y=h \nabla_{X Y} y$. Then $h \nabla_{X Y}=h \nabla_{X Y} \nabla_{Y Y}=h \nabla_{X Y} y \nabla_{Y Y} \sqsubseteq x \alpha \nabla_{Y Y}$. But $\nabla_{X Y} \sqsubseteq x \alpha \nabla_{Y Y}$ by 3.4(c) since $x \alpha \nabla_{Y Y}$ is crisp by $4.2(\mathrm{c})$. Hence $k \nabla_{X Y} \sqsubseteq k \nabla_{X Y} \alpha \nabla_{Y Y}=x(k \alpha) \nabla_{Y Y} \sqsubseteq x\left(k^{\prime} \alpha^{\prime}\right) \sqsubseteq k^{\prime} \nabla_{X Y}$. Therefore $k \leq k^{\prime}$ by 3.2(f). (e) It is trivial that if $\gamma=O_{X Y}$ then $\gamma=0 \nabla_{X Y} y$ by F2(a). Next assume $\gamma \neq 0$. Then by the point axiom there are a scalar $k>0$ and points $x_{0}, y_{0}$ such that $x_{0} \gamma \sqcap \nabla_{X Y} y_{0}=k \nabla_{X Y} y_{0}$. Note that $\gamma=\nabla_{X X} \gamma=x_{0} \nabla_{X X} \gamma=x_{0} \gamma$ by 2.3(c). Hence $k \nabla_{X Y} y_{0} \sqsubseteq x_{0} \gamma=\gamma \sqsubseteq \nabla_{X Y} y$, which implies $y_{0}=y$ by $4.3(\mathrm{~b})$ and so $\gamma=k \nabla_{X Y} y$. The uniqueness of $k$ follows from $(\mathrm{d})$.

Proposition 4.6 Let $\chi(X)$ be the set of all points of an object $X$ in $\mathcal{Z}$. Then the following holds:

(a) $\nabla_{X X}=\sqcup_{x \in \chi(X)} x$,

(b) $\operatorname{id}_{X}=\sqcup_{x \in \chi(X)} x^{\sharp} x$.

Proof. (a) Set $\alpha=\sqcup_{x \in X(X)} x$. It is clear that $\alpha \sqsubseteq \nabla_{X X}$ and $\nabla_{X X} \alpha=\alpha$. As points are crisp by the definition, $\alpha$ is also crisp by $2.2(\mathrm{~d})$ and by the axiom F3 there is a morphism $\bar{\alpha}$ such that $\alpha \sqcup \bar{\alpha}=\nabla_{X X}$ and $\alpha \sqcap \bar{\alpha}=O_{X X}$. Then $\nabla_{X X} \bar{\alpha}=\bar{\alpha}$ by 3.4(d). Assume $\bar{\alpha} \neq O_{X X}$. By the point axiom P2 there are a scalar $k>0$ and points $x_{0}, x_{1}: X \rightarrow X$ such that $x_{0} \bar{\alpha} \sqcap x_{1}=k x_{1}$. Hence $k x_{1} \sqsubseteq x_{0} \bar{\alpha} \sqsubseteq \nabla_{X X} \bar{\alpha}=\bar{\alpha}$. But $\bar{\alpha}$ is crisp by 3.4(c) and so $x_{1} \sqsubseteq \bar{\alpha}$ by 3.4(e). Therefore $x_{1} \sqsubseteq \alpha \sqcap \bar{\alpha}=O_{X X}$, which contradicts to $x_{1} \neq O_{X X}$. This proves $\bar{\alpha}=O_{X X}$. (b) It is clear that $\sqcup_{x \in \chi(X)} x^{\sharp} x \sqsubseteq \mathrm{id}_{X}$. Using $\nabla_{X X}=\sqcup_{x \in \chi(X)} x$ by (a) we have $\mathrm{id}_{X}=\mathrm{id}_{X} \sqcap \nabla_{X X}=\sqcup_{x \in \chi(X)}\left(\mathrm{id}_{X} \sqcap x\right) \sqsubseteq \sqcup_{x \in \chi(X)}\left(\mathrm{id}_{X} x^{\sharp} \sqcap \mathrm{id}_{X}\right) x \sqsubseteq \sqcup_{x \in \chi(X)} x^{\sharp} x$.

Thus from the point axiom P2 and 4.5(e) there exists a unique scalar $\chi(\alpha)(x, y)$ such that

$$
x \alpha \sqcap \nabla_{X Y} y=\chi(\alpha)(x, y) \nabla_{X Y} y .
$$

Thus $\chi(\alpha)$ defines a fuzzy relation from the set of all points of $X$ into the set of all points of $Y$. In the next section we will prove that the function $\chi: \mathcal{Z} \rightarrow \mathbf{R e l}_{[0,1]}$ is an embedding of Zadeh categories. 


\section{Representation Theorem}

First we prove the representation theorem for Zadeh categories asserting that every morphism in a Zadeh category satisfying the point axiom, can be represented as a union of pair morphisms with semi-scalar weights.

Theorem 5.1 (Representation Theorem) Every morphism $\alpha: X \rightarrow Y$ in a Zadeh category $\mathcal{Z}$ satisfying the point axiom has a unique representation

$$
\alpha=\sqcup_{x \in \chi(X)} \sqcup_{y \in \chi(Y)} \chi(\alpha)(x, y) x^{\sharp} \nabla_{X Y} y,
$$

where $\chi(\alpha)(x, y)$ is a unique scalar such that $x \alpha \sqcap \nabla_{X Y} y=\chi(\alpha)(x, y) \nabla_{X Y} y$.

Proof. Since $\nabla_{Y Y}=\sqcup_{y \in \chi(Y)} y$ by $4.6($ a) we have

$$
\begin{aligned}
x \alpha & =x \alpha \sqcap \nabla_{X Y} \\
& =x \alpha \sqcap \nabla_{X Y} \nabla_{Y Y} \\
& =x \alpha \sqcap \nabla_{X Y}\left(\sqcup_{y \in \chi(Y)} y\right) \\
& =\sqcup_{y \in \chi(Y)}\left(x \alpha \sqcap \nabla_{X Y} y\right) \\
& =\sqcup_{y \in \chi(Y) \chi(\alpha)(x, y) \nabla_{X Y} y}
\end{aligned}
$$

and so

$$
\begin{aligned}
& \alpha=\operatorname{id}_{X} \alpha \\
& =\sqcup_{x \in \chi(X)} x^{\sharp} x \alpha \\
& =\sqcup_{x \in \chi(X)} x^{\sharp}\left[\sqcup_{y \in \chi(Y) \chi}(\alpha)(x, y) \nabla_{X Y} y\right] \\
& =\sqcup_{x \in \chi(X)} \sqcup_{y \in \chi(Y)} x^{\sharp}\left[\chi(\alpha)(x, y) \nabla_{X Y} y\right] \\
& =\sqcup_{x \in \chi(X)} \sqcup_{y \in \chi(Y)} \chi(\alpha)(x, y) x^{\sharp} \nabla_{X Y} y
\end{aligned}
$$

using $\operatorname{id}_{X}=\sqcup_{x \in \chi(X)} x^{\sharp} x$ by $4.6(b)$. Finally we show the uniqueness of the representation. Assume $\alpha=\sqcup_{x \in \chi(X)} \sqcup_{y \in \chi(Y)} k_{x, y} x^{\sharp} \nabla_{X Y} y$. Then for all $x_{0} \in \chi(X), y_{0} \in \chi(Y)$ we have

$$
x_{0} \alpha=\sqcup_{x \in \chi(X)} \sqcup_{y \in \chi(Y)} k_{x, y} x_{0} x^{\sharp} \nabla_{X Y} y=\sqcup_{y \in \chi(Y)} k_{x_{0}, y} \nabla_{X Y} y
$$

and

$$
x_{0} \alpha \sqcap \nabla_{X Y} y_{0}=\sqcup_{y \in \chi(Y)}\left[k_{x_{0}, y} \nabla_{X Y} y \sqcap \nabla_{X Y} y_{0}\right]=k_{x_{0}, y_{0}} \nabla_{X Y} y_{0} .
$$

Hence $k_{x, y}=\chi(\alpha)(x, y)$ by $4.5(\mathrm{e})$.

Corollary $\mathbf{5 . 2}$ For all objects $X$ and $Y$ in $\mathcal{Z}$ the function $\chi: \mathcal{Z}(X, Y) \rightarrow \operatorname{Re}_{[0,1]}(\chi(X), \chi(Y))$ is a bijection.

Proof. If $\chi(\alpha)=\chi\left(\alpha^{\prime}\right)$, then by the last theorem we have

$$
\alpha=\sqcup_{x \in \chi(X)} \sqcup_{y \in \chi(Y)} \chi(\alpha)(x, y) x^{\sharp} \nabla_{X Y} y=\sqcup_{x \in \chi(X)} \sqcup_{y \in \chi(Y)} \chi\left(\alpha^{\prime}\right)(x, y) x^{\sharp} \nabla_{X Y} y=\alpha^{\prime},
$$

which shows that $\chi$ is injective. Given a fuzzy relation $R: \chi(X) \times \chi(Y) \rightarrow[0,1]$ we set $\alpha_{R}=\sqcup_{x \in \chi(X)} \sqcup_{y \in \chi(Y)} R(x, y) x^{\sharp} \nabla_{X Y} y$. Then by the uniqueness of representation in the last theorem we have $R(x, y)=\chi\left(\alpha_{R}\right)(x, y)$, which means that $\chi$ is surjective.

Theorem 5.3 For a morphism $\alpha$ and a set $\left\{k_{\lambda}\right\}_{\lambda}$ of scalars the identity $\sqcup_{\lambda} k_{\lambda} \alpha=\left(\vee_{\lambda} k_{\lambda}\right) \alpha$ holds. 
Proof. First we show that $\sqcup_{\lambda} k_{\lambda} x^{\sharp} \nabla_{X Y} y=\left(\vee_{\lambda} k_{\lambda}\right) x^{\sharp} \nabla_{X Y} y$ for points $x: X-X$ and $y$ : $Y \rightarrow Y$. It is obvious that $\sqcup_{\lambda} k_{\lambda} x^{\sharp} \nabla_{X Y} y \sqsubseteq\left(\vee_{\lambda} k_{\lambda}\right) x^{\sharp} \nabla_{X Y} y$ and so there is a scalar $k$ such that $\sqcup_{\lambda} k_{\lambda}\left(x^{\sharp} \nabla_{X Y} y\right)=k x^{\sharp} \nabla_{X Y} y$. Then $k \leq \vee_{\lambda} k_{\lambda}$ by $k x^{\sharp} \nabla_{X Y} y \sqsubseteq\left(\vee_{\lambda} k_{\lambda}\right) x^{\sharp} \nabla_{X Y} y$ and $4.5(\mathrm{~b})$. On the other hand $k_{\lambda} \leq k$ from $k_{\lambda} x^{\sharp} \nabla_{X Y} y \sqsubseteq k x^{\sharp} \nabla_{X Y} y$ and so $\vee_{\lambda} k_{\lambda} \leq k$. Therefore this proves $k=\vee_{\lambda} k_{\lambda}$. We are ready to prove the general case. Since $\alpha=\sqcup_{x, y \in X} \chi(\alpha)(x, y) x^{\sharp} \nabla_{X Y} y$ by the representability theorem 5.1 we have

$$
\begin{aligned}
\sqcup_{\lambda} k_{\lambda} \alpha & =\sqcup_{\lambda} k_{\lambda}\left[\sqcup_{x \in \chi(X)} \sqcup_{y \in \chi(Y)} \chi(\alpha)(x, y) x^{\sharp} \nabla_{X Y} y\right] \\
& =\sqcup_{\lambda} \sqcup_{x \in \chi(X)} \sqcup_{y \in \chi(Y)}\left[k_{\lambda} \chi(\alpha)(x, y) x^{\sharp} \nabla_{X Y} y\right] \\
& =\sqcup_{x \in \chi(X)} \sqcup_{y \in \chi(Y)} \sqcup_{\lambda}\left[k_{\lambda} \chi(\alpha)(x, y) x^{\sharp} \nabla_{X Y} y\right] \\
& =\sqcup_{x \in \chi(X)} \sqcup_{y \in \chi(Y)}\left[\left(\vee_{\lambda} k_{\lambda}\right) \chi(\alpha)(x, y) x^{\sharp} \nabla_{X Y} y\right] \\
& =\left(\vee_{\lambda} k_{\lambda}\right)\left[\sqcup_{x \in \chi(X)} \sqcup_{\left.y \in \chi(Y) \chi(\alpha)(x, y) x^{\sharp} \nabla_{X Y} y\right]}\right. \\
& =\left(\vee_{\lambda} k_{\lambda}\right) \alpha .
\end{aligned}
$$

The following theorem is known as Tarski rule for Boolean morphism algebras $[13,15]$.

Theorem 5.4 For a nonzero morphism $\alpha: X \rightarrow Y$ in $\mathcal{Z}$ there is a scalar $k>0$ such that $\nabla_{X X} \alpha \nabla_{Y Y}=k \nabla_{X Y}$.

Proof. By means of the representation theorem 5.1 we have

$$
\begin{aligned}
\nabla_{X X} \alpha \nabla_{Y Y} & =\sqcup_{x \in \chi(X)} \sqcup_{y \in \chi(Y)} \nabla_{X X}\left[\chi(\alpha)(x, y) x^{\sharp} \nabla_{X Y} y\right] \nabla_{Y Y} \\
& =\sqcup_{x \in \chi(X)} \sqcup_{y \in \chi(Y)} \chi(\alpha)(x, y)\left[\nabla_{X X} x^{\sharp} \nabla_{X Y} y \nabla_{Y Y}\right] \\
& =\sqcup_{x \in \chi(X)} \sqcup_{y \in \chi(Y)} \chi(\alpha)(x, y) \nabla_{X X} \nabla_{X Y} \nabla_{Y Y} \\
& =\sqcup_{x \in \chi(X)} \sqcup_{y \in \chi(Y) \chi(\alpha)(x, y) \nabla_{X Y}} \\
& =\left[\vee_{x \in \chi(X)} \vee_{y \in \chi(Y) \chi(\alpha)(x, y)] \nabla_{X Y} .}\right.
\end{aligned}
$$

Set $k=\vee_{x \in \chi(X)} \vee_{y \in \chi(Y)} \chi(\alpha)(x, y)$. Then $\nabla_{X X} \alpha \nabla_{Y Y}=k \nabla_{X Y}$. By the way there exist points $x, y$ such that $\chi(\alpha)(x, y)>0$ by the point axiom P2. Hence $k>0$, which completes the proof.

The following proposition shows that $\chi: \mathcal{Z} \rightarrow \mathbf{R e l}_{[0,1]}$ preserves all operations of fuzzy morphisms, that is, $\chi$ is a homomorphism of fuzzy relation algebras.

Proposition 5.5 Let $\alpha, \alpha^{\prime}: X \rightarrow Y, \beta: Y \rightarrow Z$ be morphisms and $k$ a scalar. Then the following holds :

(a) $\chi\left(O_{X Y}\right)=O_{\chi(X) \chi(Y)}, \chi\left(\nabla_{X Y}\right)=\nabla_{\chi(X) \chi(Y)}$ and $\chi\left(\mathrm{id}_{X}\right)=\mathrm{id}_{\chi(X)}$.

(b) $\chi\left(\alpha \sqcup \alpha^{\prime}\right)=\chi(\alpha) \cup \chi\left(\alpha^{\prime}\right)$.

(c) $\chi\left(\alpha \sqcap \alpha^{\prime}\right)=\chi(\alpha) \cap \chi\left(\alpha^{\prime}\right)$.

(d) $\chi\left(\alpha^{\sharp}\right)=\chi(\alpha)^{\sharp}$.

(e) $\chi(k \alpha)=k \chi(\alpha)$.

(f) $\chi(\alpha \beta)=\chi(\alpha) \chi(\beta)$.

Proof. (a) The fist follows from $x O_{X Y} \sqcap \nabla_{X Y} y=O_{X Y}=0 y$, the second follows from $x \nabla_{X Y} \sqcap$ $\nabla_{X Y} y=\nabla_{X Y} \sqcap \nabla_{X Y} y=\nabla_{X Y} y$ by $x \nabla_{X Y}=x \nabla_{X X} \nabla_{X Y}=\nabla_{X X} \nabla_{X Y}=\nabla_{X Y}$, and the last 
follows from $x \operatorname{id}_{X} \sqcap \nabla_{X X} x^{\prime}=x \sqcap x^{\prime}$ and $4.5(\mathrm{e})$.

(b) It follows from

$$
\begin{aligned}
\chi(\alpha \sqcup \beta)(x, y) \nabla_{X Y} y & =x(\alpha \sqcup \beta) \sqcap \nabla_{X Y} y \\
& =(x \alpha \sqcup x \beta) \sqcap \nabla_{X Y} y \\
& =\left(x \alpha \sqcap \nabla_{X Y} y\right) \sqcup\left(x \beta \sqcap \nabla_{X Y} y\right) \\
& =\chi(\alpha)(x, y) \nabla_{X Y} y \sqcup \chi(\beta)(x, y) \nabla_{X Y} y \\
& =[\chi(\alpha)(x, y) \vee \chi(\beta)(x, y)] \nabla_{X Y} y \\
& =[\chi(\alpha) \cup \chi(\beta)](x, y) \nabla_{X Y} y .
\end{aligned}
$$

(c) First note that $x\left(\alpha \sqcap \alpha^{\prime}\right)=x \alpha \sqcap x \alpha^{\prime}$. It follows from $x^{\sharp} x \sqsubseteq \mathrm{id} x$ that $x\left(\alpha \sqcap \alpha^{\prime}\right) \sqsubseteq x \alpha \sqcap x \alpha^{\prime} \sqsubseteq$ $x\left(\alpha \sqcap x^{\sharp} x \alpha^{\prime}\right) \sqsubseteq x\left(\alpha \sqcap \alpha^{\prime}\right)$. Hence we have

$$
\begin{aligned}
\chi\left(\alpha \sqcap \alpha^{\prime}\right)(x, y) \nabla_{X Y} y & =x\left(\alpha \sqcap \alpha^{\prime}\right) \sqcap \nabla_{X Y} y \\
& =\left(x \alpha \sqcap x \alpha^{\prime}\right) \sqcap \nabla_{X Y} y \\
& =\left(x \alpha \sqcap \nabla_{X Y} y\right) \sqcap\left(x \alpha^{\prime} \sqcap \nabla_{X Y} y\right) \\
& =\chi(\alpha)(x, y) \nabla_{X Y} y \sqcap \chi\left(\alpha^{\prime}\right)(x, y) \nabla_{X Y} y \\
& =\left[\chi(\alpha)(x, y) \wedge \chi\left(\alpha^{\prime}\right)(x, y)\right] \nabla_{X Y} y \\
& =\left[\chi(\alpha) \sqcap \chi\left(\alpha^{\prime}\right)\right](x, y) \nabla_{X Y} y .
\end{aligned}
$$

(d) $\alpha \sqcap x^{\sharp} \nabla_{X Y} y=k x^{\sharp} \nabla_{X Y} y$ if and only if $x \alpha \sqcap \nabla_{X Y} y=k \nabla_{X Y} y$.

First note that $x \alpha \sqcap \nabla_{X Y} y=x\left(\alpha \sqcap x^{\sharp} \nabla_{X Y} y\right)$ and $\alpha \sqcap x^{\sharp} \nabla_{X Y} y=x^{\sharp}\left(x \alpha \sqcap \nabla_{X Y} y\right)$, because $x \alpha \sqcap \nabla_{X Y} y \sqsubseteq x\left(\alpha \sqcap x^{\sharp} \nabla_{X Y} y\right) \sqsubseteq x x^{\sharp}\left(x \alpha \sqcap \nabla_{X Y} y\right) \sqsubseteq \nabla\left(x \alpha \sqcap \nabla_{X Y} y\right) \sqsubseteq x \alpha \sqcap \nabla_{X Y} y$, and $\alpha \sqcap x^{\sharp} \nabla_{X Y} y \sqsubseteq x^{\sharp}\left(x \alpha \sqcap \nabla_{X Y} y\right) \sqsubseteq x^{\sharp} x\left(\alpha \sqcap x^{\sharp} \nabla_{X Y} y\right) \sqsubseteq \alpha \sqcap x^{\sharp} \nabla_{X Y} y$ by $x^{\sharp} x \sqsubseteq \mathrm{id}_{X}$. Now assume $\alpha \sqcap x^{\sharp} \nabla_{X Y} y=k x^{\sharp} \nabla_{X Y} y$. Then $x \alpha \sqcap \nabla_{X Y} y=x\left(\alpha \sqcap x^{\sharp} \nabla_{X Y} y\right)=x\left[k\left(x^{\sharp} \nabla_{X Y} y\right)\right]=$ $k\left[x\left(x^{\sharp} \nabla_{X Y} y\right)\right]=k \nabla_{X Y} y$. Conversely assume $x \alpha \sqcap \nabla_{X Y} y=k \nabla_{X Y} y$. Then $\alpha \sqcap x^{\sharp} \nabla_{X Y} y=$ $x^{\sharp}\left(x \alpha \sqcap \nabla_{X Y} y\right)=x^{\sharp}\left(k \nabla_{X Y} y\right)=k x^{\sharp} \nabla_{X Y} y$ (since $x^{\sharp}$ is crisp).

An identity $\chi\left(\alpha^{\sharp}\right)=\chi(\alpha)^{\sharp}$ follows from

$$
\begin{aligned}
x \alpha \sqcap \nabla_{X Y} y=k \nabla_{X Y} y & \Longleftrightarrow \alpha \sqcap x^{\sharp} \nabla_{X Y} y=k x^{\sharp} \nabla_{X Y} y \\
& \Longleftrightarrow \alpha^{\sharp} \sqcap y^{\sharp} \nabla_{Y X} x=k y^{\sharp} \nabla_{Y X} x \\
& \Longleftrightarrow y \alpha^{\sharp} \sqcap \nabla_{Y X} x=k \nabla_{Y X} x .
\end{aligned}
$$

(e) It follows from

$$
\begin{aligned}
\chi(k \alpha)(x, y) \nabla_{X Y} y & =x(k \alpha) \sqcap \nabla_{X Y} y \\
& =k(x \alpha) \sqcap k \nabla_{X Y} \sqcap \nabla_{X Y} y \quad\left(k(x \alpha) \sqsubseteq k \nabla_{X Y}\right) \\
& =k(x \alpha) \sqcap k \nabla_{X Y} y \\
& =k\left(x \alpha \sqcap \nabla_{X Y} y\right) \\
& =k\left[\chi(\alpha)(x, y) \nabla_{X Y} y\right] \\
& =[k \chi(\alpha)(x, y)] \nabla_{X Y} y \\
& =[k \chi(\alpha)](x, y) \nabla_{X Y} y .
\end{aligned}
$$

(f) When $\nabla_{Z Z}=O_{Z Z}$, it is trivial that the equality $\chi(\alpha \beta)=\chi(\alpha) \chi(\beta)$. Therefore we may assume that $Z$ is nonempty. First note that $\chi(\alpha \beta)(x, z)=\vee_{y} \chi\left(\alpha y^{\sharp} y \beta\right)(x, z)$ from

$$
\begin{aligned}
\chi(\alpha \beta)(x, z) \nabla_{X Z} z & =x \alpha \beta \sqcap \nabla_{X Z Z} \\
& =x \alpha i d_{Y} \beta \sqcap \nabla_{X Z Z} \\
& =x \alpha\left(\sqcup_{y} y^{\sharp} y\right) \beta \sqcap \nabla_{X Z} z \\
& =\sqcup_{y}\left(x \alpha y^{\sharp} y \sqcap \nabla_{X Z} z\right) \\
& =\sqcup_{y} \chi\left(\alpha y^{\sharp} y \beta\right)(x, z) \nabla_{X} z \\
& =\left[\vee_{y} \chi\left(\alpha y^{\sharp} y \beta\right)(x, z)\right] \nabla_{X Z} z . \quad \text { (by 5.3) }
\end{aligned}
$$


To complete the proof it suffices to show $\chi\left(\alpha y^{\sharp} y \beta\right)(x, z)=\chi(\alpha)(x, y) \wedge \chi(\beta)(y, z)$. Note that $x \alpha \sqcap \nabla_{X Y} y=x \alpha y^{\sharp} y$ from $x \alpha y^{\sharp} y \sqsubseteq x \alpha \sqcap \nabla_{X Y} y \sqsubseteq\left(x \alpha y^{\sharp} \sqcap \nabla_{X Y}\right) y=x \alpha y^{\sharp} y$, and $\nabla_{X Z}\left(z \beta^{\sharp} \sqcap\right.$ $\left.\nabla_{Z Y} y\right)=\nabla_{X Z} z \beta^{\sharp} \sqcap \nabla_{X Y} y$ from $\nabla_{X Z}\left(z \beta^{\sharp} \sqcap \nabla_{Z Y} y\right) \sqsubseteq \nabla_{X Z} z \beta^{\sharp} \sqcap \nabla_{X Z} \nabla_{Z Y} y \sqsubseteq \nabla_{X Z} z \beta^{\sharp} \sqcap \nabla_{X Y} y \sqsubseteq$ $\nabla_{X Z}\left(z \beta^{\sharp} \sqcap \nabla_{Z X} \nabla_{X Y} y\right) \sqsubseteq \nabla_{X Z}\left(z \beta^{\sharp} \sqcap \nabla_{Z Y} y\right)$. Then we have

$$
\begin{aligned}
{[\chi(\alpha)(x, z) \wedge \chi(\beta)(y, z)] \nabla_{X Y} y } & =\chi(\alpha)(x, y) \nabla_{X Y} y \sqcap \chi(\beta)(y, z) \nabla_{X Y} y \quad(\text { by } \mathrm{F} 2(\mathrm{~d})) \\
& =\chi(\alpha)(x, y) \nabla_{X Y} y \sqcap \chi\left(\beta^{\sharp}\right)(y, z) \nabla_{X Z} \nabla_{Z Y} y \\
& =\left(x \alpha \sqcap \nabla_{X Y} y\right) \sqcap \nabla_{X Z}\left(z \beta^{\sharp} \sqcap \nabla_{Z Y} y\right) \\
& =\left(x \alpha \sqcap \nabla_{X Y} y\right) \sqcap\left(\nabla_{X Z} z \beta^{\sharp} \sqcap \nabla_{X Y} y\right) \\
& =x \alpha y^{\sharp} y \sqcap \nabla_{X Z} z \beta^{\sharp} .
\end{aligned}
$$

Set $r=\chi\left(\alpha y^{\sharp} y \beta\right)(x, z)$ and $s=\chi(\alpha)(x, y) \wedge \chi(\beta)(y, z)$. Then $x \alpha y^{\sharp} y \beta \sqcap \nabla_{X Z} z=r \nabla_{X Z} z$ and $x \alpha y^{\sharp} y \sqcap \nabla_{X Z} z \beta^{\sharp}=s \nabla_{X Y} y$. We have $r \leq s$ from

$$
r \nabla_{X Z} z=x \alpha y^{\sharp} y \beta \sqcap \nabla_{X Z} z \sqsubseteq\left(x \alpha y^{\sharp} y \sqcap \nabla_{X Z} z \beta^{\sharp}\right) \beta \sqsubseteq\left(s \nabla_{X Y} y\right) \beta \sqsubseteq s \nabla_{X Z},
$$

and $s \leq r$ from

$$
s \nabla_{X Y} y=x \alpha y^{\sharp} y \sqcap \nabla_{X Z} z \beta^{\sharp} \sqsubseteq\left(x \alpha z^{\sharp} z \beta \sqcap \nabla_{X Z} z\right) \beta^{\sharp} \sqsubseteq\left(r \nabla_{X Z} z\right) \beta^{\sharp} \sqsubseteq r \nabla_{X Y} .
$$

Therefore $r=s$.

The following is immediately deduced from the last proposition.

Corollary 5.6 (Insertion Theorem) Let $\alpha: X \rightarrow Y$ and $\beta: Y \rightarrow Z$ be morphisms and let $x: X \rightarrow X$ and $z: Z \rightarrow Z$ be points. If $\chi(\alpha \beta)(x, z)>0$, then there is a point $y: Y \rightarrow Y$ such that $\chi(\alpha)(x, y)>0$ and $\chi(\beta)(y, z)>0$.

Proposition 5.7 Let $\mathcal{Z}$ be a Zadeh category satisfying the point axiom and $I$ a unit object in $\mathcal{Z}$.

(a) If $u: I-I$ is a morphism, then there is a scalar $k$ such that $u=k \operatorname{id}_{I}$.

(b) A morphism $\tau: I \rightarrow X$ is crisp if and only if it is I-crisp.

(c) The unit object I satisfies the strict point axiom.

(d) $\xi(\alpha)\left(\nabla_{I X} x, \nabla_{I Y} y\right)=\chi(\alpha)(x, y) \mathrm{id}_{I}$ for $I$-points $x$ of $X$ and $y$ of $Y$.

Proof. (a) Recall that $\mathrm{id}_{I}=\nabla_{I I}$ and a unit object $I$ has just one point $\mathrm{id}_{I}$. If $u$ is nonzero, then by the point axiom $\mathrm{P} 1$ we have $u \sqcap \mathrm{id}{ }_{I}=k \mathrm{id}_{I}$ for a scalar $k>0$. (b) First we show that a crisp morphism $\tau: I \rightarrow X$ is $I$-crisp. Assume that $\tau$ is crisp and $u \tau^{\prime} \sqsubseteq \tau$ for $\tau^{\prime}: I-X$ and $u \neq O_{I I}: I-I$. By (a) there is a scalar $k>0$ such that $u=k \operatorname{id}_{I}$ and so $k \tau^{\prime}=k\left(\mathrm{id}_{I} \tau^{\prime}\right)=$ $\left(k \operatorname{id}_{I}\right)\left(k \tau^{\prime}\right) \sqsubseteq u \tau^{\prime} \sqsubseteq \tau$. As $\tau$ is crisp we have $\tau^{\prime} \sqsubseteq \tau$ by 3.4(c), which indicates that $\tau$ is $I$-crisp. Conversely we show that an $I$-crisp morphism is crisp. Assume that $\tau: I \rightarrow X$ is $I$-crisp. Note that $\tau=\tau \operatorname{id}_{X}=\sqcup_{x \in \chi(X)} \tau x^{\sharp} x$ by 4.6(b) If $u=\tau x^{\sharp}$ is nonzero, then $x \sqsubseteq \tau$ because $\tau$ is $I$-crisp, and so $u=\mathrm{id}_{I}$. Hence $\tau=\sqcup_{x \sqsubseteq \tau} x$ and consequently $\tau$ is crisp by $3.4(\mathrm{a})$.

(c) Let $x: X-X$ be a point of $X$ and set $p=\nabla_{I X} x$. Then $p^{\sharp} p=x^{\sharp} \nabla_{X I} \nabla_{I X} x \sqsubseteq x^{\sharp} \nabla_{X X} x=$ $x^{\sharp} x \sqsubseteq \mathrm{id}_{X}, \mathrm{id}_{X}=\nabla_{I I}=\nabla_{I X} \nabla_{X I} \sqsubseteq \nabla_{I X} x x^{\sharp} \nabla_{X I}=p p^{\sharp}$ (since $X$ has a point $x$ ). As $x$ and $\nabla_{I X}$ are crisp, $p$ is crisp by $4.2(\mathrm{c})$ and so $p$ is $I$-crisp by (b). This proves that $p=\nabla_{I X} x$ is an $I$-point of $X$. Hence $\nabla_{I X}=\nabla_{I X} \mathrm{id}_{X}=\nabla_{I X}\left(\sqcup_{x \in \chi(X)} x^{\sharp} x\right)=\sqcup_{x \in \chi(X)} \nabla_{I X} x^{\sharp} x \sqsubseteq \sqcup_{x \in \chi(X)} \nabla_{I X} x \sqsubseteq$ 
$\sqcup_{p \in \xi(X)} p$, which means $\nabla_{I X}=\sqcup_{p \in \xi(X)} p$.

(d) Set $k=\chi(\alpha)(x, y)$. As has been seen in (c) $\nabla_{I X} x$ and $\nabla_{I Y} y$ are $I$-points and so we have

$$
\begin{aligned}
\xi(\alpha)\left(\nabla_{I X} x, \nabla_{I Y} y\right) & =\nabla_{I X} x \alpha\left(\nabla_{I Y} y\right)^{\sharp} \\
& =\nabla_{I X}\left(x \alpha y^{\sharp} \sqcap \nabla_{X Y}\right) \nabla_{Y I} \\
& =\nabla_{I X}\left(x \alpha y^{\sharp} \sqcap \nabla_{X Y} y y^{\sharp}\right) \nabla_{Y I} \\
& =\nabla_{I X}\left(x \alpha \sqcap \nabla_{X Y} y\right) y^{\sharp} \nabla_{Y I} \\
& =\nabla_{I X}\left(k \nabla_{X Y} y\right) y^{\sharp} \nabla_{Y I} \\
& =k \nabla_{I X} \nabla_{X Y} y y^{\sharp} \nabla_{Y I} \\
& =k \nabla_{I X} \nabla_{X Y} \nabla_{Y I} \\
& =k \operatorname{id}_{I} . \square
\end{aligned}
$$

Consequently we have proved that a Zadeh category satisfying the point axiom is equivalent to a subcategory of the category of sets and (ordinary) fuzzy relations. It is an open problem whether a Dedekind category $\mathcal{D}$ with a unit object $I$ satisfying the strict point axiom and with $\mathcal{D}(I, I) \cong[0,1]$ is a Zadeh category.

\section{References}

[1] P. Freyd and A. Scedrov, Categories, allegories (North-Holland, Amsterdam, 1990).

[2] J.A. Goguen, L-fuzzy set, J. Math. Anal. Appl. 18 (1967) 145-174.

[3] Y. Kawahara, Pushout-complements and basic concepts of grammars in topoi, Theoretical Computer Science 77 (1990) 267-289.

[4] Y. Kawahara, Relational set theory, Lecture Notes in Computer Science, 953(1995) 44-58.

[5] Y. Kawahara and H. Furusawa, An algebraic formalization of fuzzy relations, RIFIS-TRCS 98, Kyushu University, February 1995.

[6] Y. Kawahara and Y. Mizoguchi, Relational structures and their partial morphisms in the view of single pushout rewriting, Lecture Notes in Computer Science 776 (1994) 218-233.

[7] S. Mac Lane, An algebra of additive relations, Proc. Nat. Acad. Sci. U.S.A. 47(1961) $1043-1051$.

[8] R.D. Maddux, The origin of relation algebras in the development and axiomatization of the calculus of relations, Studia Logica, 50 (1991) 423-455.

[9] Jean-Pierre Olivier and Dany Serrato, Squares and rectangles in relation categories Three cases : semilattice, distributive lattice and boolean non-unitary, Fuzzy Sets and Systems 72 (1995) 167-178.

[10] W. Pedrycz, Processing in relational structures: Fuzzy relational equations, Fuzzy Sets and Systems 40 (1991) 77-106.

[11] D. Puppe, Korrespondenzen in Abelschen Kategorien, Math. Ann. 148 (1962) 1-30.

[12] E. Sanchez, Resolution of composite fuzzy relation equations, Information and Control 30 (1976) 38-48.

[13] G. Schmidt and T. Ströhlein, Relation algebras : Concept of points and representability, Discrete Mathematics 54 (1985) 83-92. 
[14] G. Schmidt and T. Ströhlein, Relations and graphs - Discrete Mathematics for Computer Science - (Springer-Verlag, Berlin, 1993).

[15] A. Tarski, On the calculus of relations, J. Symbolic Logic 6 (1941) 73-89. 